\title{
Calretinin-positive L5a pyramidal neurons in the development of the paralemniscal pathway in the barrel cortex
}

Junhua Liu', Bin Liu', XiaoYun Zhang ${ }^{1}$, Baocong Yu', Wuqiang Guan ${ }^{3}$, Kun Wang ${ }^{1}$, Yang Yang ${ }^{1}$, Yifan Gong ${ }^{1}$, Xiaojing $\mathrm{Wu}^{1}$, Yuchio Yanagawa ${ }^{4}$, Shengxi $\mathrm{Wu}^{5}$ and Chunjie Zhao ${ }^{1,2^{*}}$

\begin{abstract}
Background: The rodent barrel cortex has been established as an ideal model for studying the development and plasticity of a neuronal circuit. The barrel cortex consists of barrel and septa columns, which receive various input signals through distinct pathways. The lemniscal pathway transmits whisker-specific signals to homologous barrel columns, and the paralemniscal pathway transmits multi-whisker signals to both barrel and septa columns. The integration of information from both lemniscal and paralemniscal pathways in the barrel cortex is critical for precise object recognition. As the main target of the posterior medial nucleus (POm) in the paralemniscal pathway, layer 5a (L5a) pyramidal neurons are involved in both barrel and septa circuits and are considered an important site of information integration. However, information on $L 5 a$ neurons is very limited. This study aims to explore the cellular features of $L 5 a$ neurons and to provide a morphological basis for studying their roles in the development of the paralemniscal pathway and in information integration.
\end{abstract}

Results: 1. We found that the calcium-binding protein calretinin (CR) is dynamically expressed in L5a excitatory pyramidal neurons of the barrel cortex, and L5a neurons form a unique serrated pattern similar to the distributions of their presynaptic POm axon terminals.

2. Infraorbital nerve transection disrupts this unique alignment, indicating that it is input dependent.

3. The formation of the L5a neuronal alignment develops synchronously with barrels, which suggests that the lemniscal and paralemniscal pathways may interact with each other to regulate pattern formation and refinement in the barrel cortex.

4. CR is specifically expressed in the paralemniscal pathway, and CR deletion disrupts the unique L5a neuronal pattern, which indicates that CR may be required for the development of the paralemniscal pathway.

Conclusions: Our results demonstrate that L5a neurons form a unique, input-dependent serrated alignment during the development of cortical barrels and that CR may play an important role in the development of the paralemniscal pathway. Our data provide a morphological basis for studying the role of L5a pyramidal neurons in information integration within the lemniscal and paralemniscal pathways.

Keywords: Calretinin, L5a pyramidal neuron, Paralemniscal pathway, Posterior medial nucleus, Barrel cortex

\footnotetext{
* Correspondence: zhaocj@seu.edu.cn

${ }^{1}$ Key Laboratory of Developmental Genes and Human Diseases, MOE,

Department of Anatomy and Neuroscience, Medical School, Southeast

University, Nanjing 210009, PR China

${ }^{2}$ Center of Depression, Beijing Institute for Brain Disorders, Beijing 100069,

PR China

Full list of author information is available at the end of the article
} 


\section{Background}

In rodents, the primary somatosensory cortex (barrel cortex) integrates into the barrel- and septum-related columnar circuits and receives distinct sensory inputs via the lemniscal and the paralemniscal pathways from the whiskers [1,2]. Via a relay of the principal sensory trigeminal nucleus (Pr5) and the contralateral ventroposteromedial nucleus (VPM), the lemniscal pathway transmits whisker-specific information exclusively to the barrel circuit and forms an input-dependent barrel pattern from the periphery to the center [3]. Likewise, through the spinal trigeminal nucleus $(\mathrm{Sp} 5 \mathrm{i})$ and the contralateral posterior medial nucleus (POm), the paralemniscal pathway transmits information from multiple whiskers into both barrel and septa circuits $[1,2,4,5]$. The main targets of POm projections are L5a neurons, although the axons also terminate at layer 4 (L4) septa and in L1. It is known that presynaptic POm axon terminals form a distinct pattern in which more axons project to the areas of L5a under septa than to the neighboring areas under barrels, and these terminals display regularly spaced triangular structures in L5A septa columns $[1,2,6,7]$. However, whether L5a neurons are organized into unique patterns in the paralemniscal pathway, similar to those of L4 neurons in the lemniscal pathway, has not been clearly demonstrated.

In the barrel cortex, L5a is involved in both barrel and septa circuits and is considered to be an important site of information integration for the lemniscal and paralemniscal pathways $[4,8]$. Electrophysiological studies show that L5a pyramidal neurons are exclusively activated by input from the POm rather than the VPM [4] and can form various types of intracortical synaptic connections with layer $2 / 3$ (L2/3) neurons, L4 spiny satellite cells, and local L5a pyramidal neurons [9-11]. These studies have all indicated that L5a pyramidal neurons play key roles in barrel and septa circuits $[4,12]$. However, to date, L5a pyramidal neurons are mainly distinguished by their approximate positions or by neuronal tracing, and the morphology of L5a pyramidal neurons remains poorly understood, which has prevented further clarification of the roles of L5a pyramidal neurons in the integration of information.

In this study, we found that the calcium binding protein calretinin (CR) is dynamically expressed in L5a pyramidal neurons of the barrel cortex. L5a pyramidal neurons form a unique, predominantly serrated pattern that is quite similar to the presynaptic projection pattern formed the POm in L5a. This unique alignment is dependent on sensory input, and its organization is synchronous with barrel formation during brain development. Furthermore, deletion of CR disrupts this alignment. In addition to L5a pyramidal neurons, CR is also expressed in the POm and Sp5i of the paralemniscal pathway during development, which indicates that $C R$ may be involved in the development of the paralemniscal pathway. Our results provide an important morphological basis for studying the functions of L5a pyramidal neurons during paralemniscal pathway development and information integration.

\section{Results}

\section{CR is dynamically expressed in L5a pyramidal neurons of} the barrel cortex

$\mathrm{CR}$, a calcium-binding protein, is reported to be an important modulator of neuronal excitability [13], with involvement in synaptogenesis, axonal elongation and dendritic remodeling $[13,14]$. Here, we detected the postnatal expression and possible role of $\mathrm{CR}$ in the mouse somatosensory cortex. At birth, CR was scarce in the somatosensory cortex (Figure 1A). At P3, a row-like band of CR expression could be detected in the deep layer of the barrel cortex (Figure 1B), and the expression level of CR gradually increased until it peaked at P8 (Figure 1D), as previously reported [14]. Scattered CR-positive neurons throughout the cortex were also observed (Figure 1A-F), and these neurons are likely interneurons according to previous reports $[15,16]$. We further found that neurons that strongly expressed CR in the row-like zone within the deeper layer of the barrel cortex are horizontally aligned in a serrated pattern (Figure 1C-D). After P8, the expression level of CR then gradually decreased and barely detectable at P30, except in scattered interneurons (Figure 1E-F). This dynamic expression of $\mathrm{CR}$ in the deeper layer of the barrel cortex is consistent with the time window during which cortical barrels develop, which suggests a possible role for CR in barrel cortex development. To identify the features and functions of the CR-positive cells, we first performed a BrdU birth-dating analysis. BrdU was injected at E12.5, E13.5, E14.5, E15.5, respectively, corresponding to the time points when layer 6 (L6), L5 and upper layer neurons are born. The brains were then harvested at P8, when the expression of $\mathrm{CR}$ is strongest in the deeper layer in the barrel cortex and almost all of the projection neurons had migrated to their final destinations in the cortical plate. Double immunostaining for $\mathrm{CR}$ and BrdU revealed that approximately $43.40 \pm 2.70 \%$ of neurons with strong CR expression in the row-like zone were also positive for BrdU administered at E13.5, which is the time point when the majority of L5 neurons are generated (Figure 1G,H,I, arrow and insert). But few of the strongly CR-positive neurons were positive for BrdU labeling applied at other time points (Figure 1J,K,L), suggesting that CR-positive neurons are mainly born at E13.5.

In the barrel cortex, L5 consists of two populations of L5a and L5b neurons that receive paralemniscal and lemniscal projections, respectively. We further determined the exact localization of CR-positive neurons within L5 at P8. When slices were co-labeled with Cux1, an upper layer (L2/3 and L4) excitatory neuronal marker, and Ctip2, a 

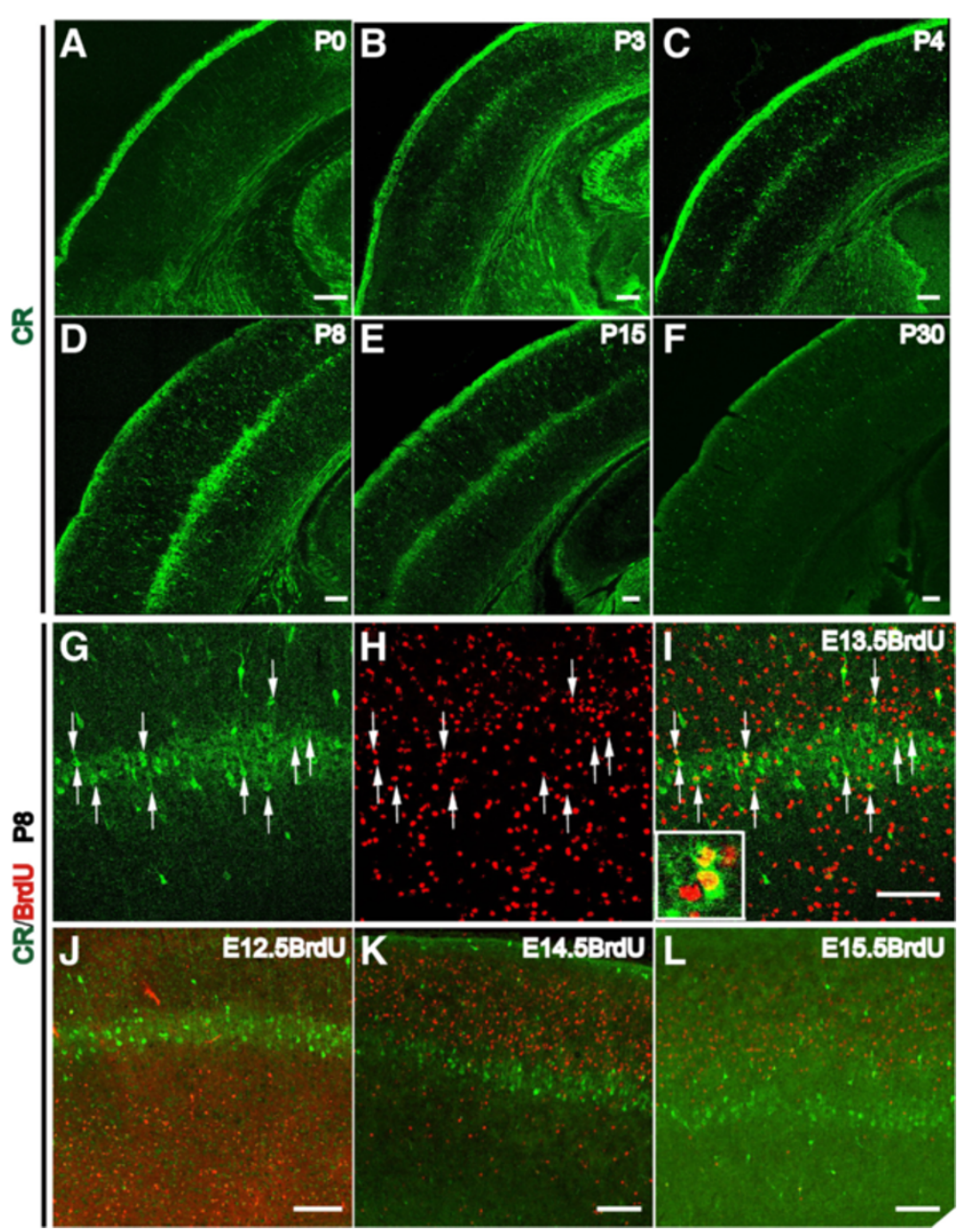

Figure $1 \mathrm{CR}$ is dynamically expressed in layer 5 of the barrel cortex during the first postnatal weeks. At birth, no CR staining can be observed in the deeper layer of the cortex (A). Beginning at P3, CR is detected in the deeper layer of the barrel cortex (B). This CR expression gradually increases and forms a clear row-like zone at P4 (C). The expression level of CR peaks at P8 (D). The intensity of the row-like zone of CR staining decreases at P15 (E). By P30, the CR-positive row-like zone can no longer be detected in the deep layer of the cortex, and the only signal comes from CR-positive interneurons dispersed throughout the cortex (F). Birth-dating analysis indicates that abundant CR-positive neurons at P8 are found to be positive for BrdU administered at E13.5 (G, H, I, arrows). Few BrdU- and CR-positive neurons were observed when BrdU was injected at E12.5 (J), E14.5 (K) and E15.5 (L), which corresponds to the time points at which L6, L4 and L2/3 neurons, respectively, are born. Scale bar: $100 \mu \mathrm{m}$.

marker for L5b excitatory neurons $[17,18]$, CR-positive neurons were found between the layers of the Cux1positive and L5b Ctip2-positive neurons without colocalization with either marker (Figure 2A-F'). Meanwhile, two transcription factors that are widely expressed in cortical excitatory neurons, neurogenic differentiation 2 (NeuroD2) [19] and forkhead box proteins 2 (Foxp2) [20], were found to be expressed in most of the CR-positive neurons (Figure 2G-L'). Statistical analysis revealed that in the row-like zone, $96.22 \pm 2.94 \%$ of CR-positive neurons were NeuroD2-positive and $97.04 \pm 2.55 \%$ were Foxp2positve. Taken together, our data indicate that CR-positive neurons are L5a excitatory pyramidal neurons, the main target of POm projections [4].
To exclude the possibility that CR-positive neurons in L5a are interneurons, double immunostaining for anti$\mathrm{CR}$ and anti-gamma-aminobutyric acid (anti-GABA) was performed. Within the row-like zone, no CRpositive neurons were found to also be GABA-positive, although CR- and GABA-positive interneurons were found scattered throughout the cortex (Figure $3 \mathrm{C}, \mathrm{C}^{\prime}$ ). This result was also confirmed by co-labeling CR with anti-GFP using the GAD67-GFP knock-in mouse line in which GFP is expressed in almost all GABAergic interneurons under the control of the endogenous GAD67 promoter [21]. Abundant GFP-positive interneurons were detected throughout the cortex, but few GFP-positive interneurons within L5a were found to also be CR-positive 


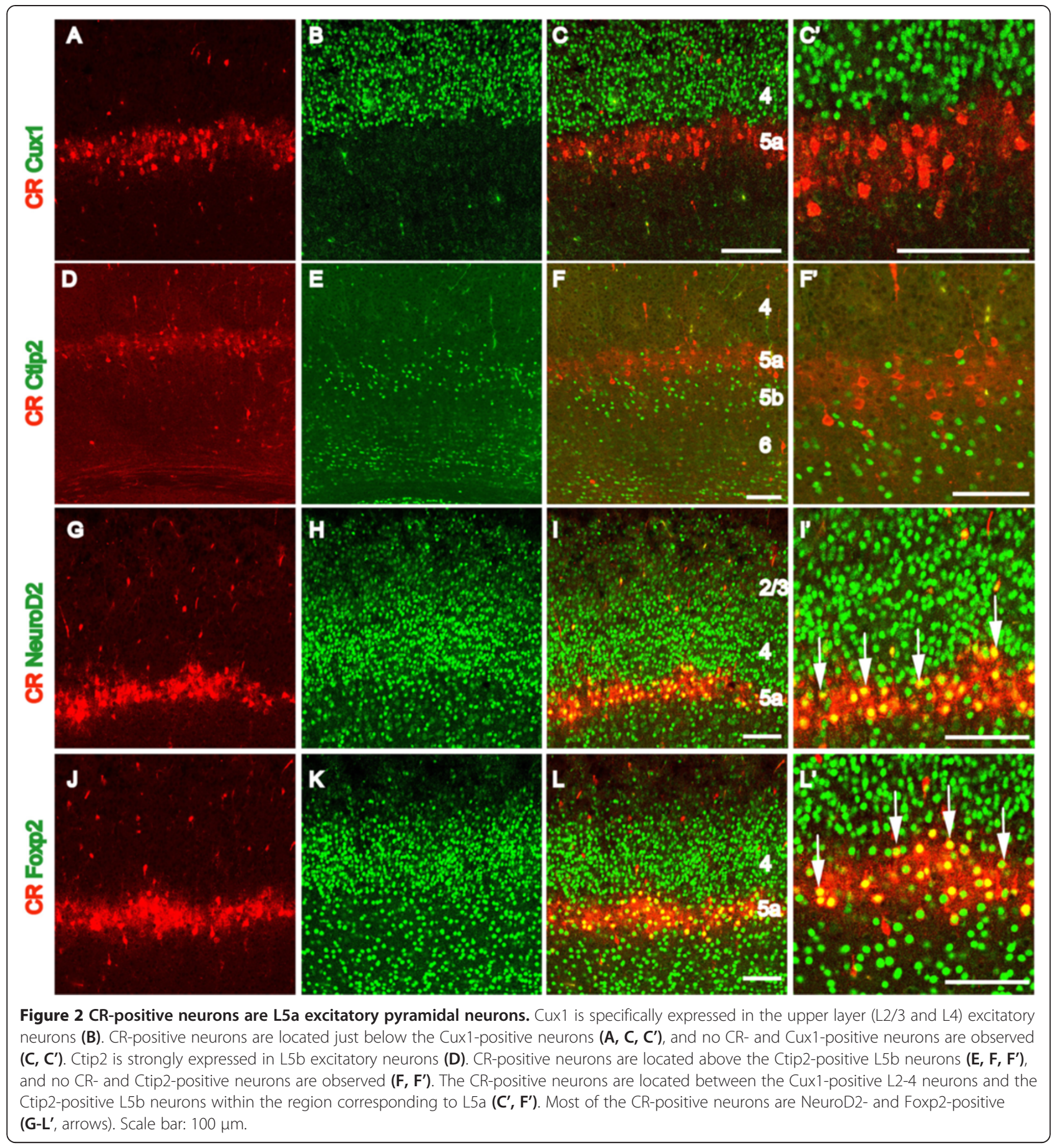

(Figure 3F,F'). These data all indicate that CR-positive neurons within L5a are excitatory pyramidal neurons and not GABAergic interneurons.

L5a pyramidal neurons form a unique serrated pattern that requires sensory input during early postnatal days for maintenance

In the barrel cortex, L5a pyramidal neurons specifically receive inputs from the POm $[1,2,4,7]$, whose axon terminals display a row-like pattern with regularly spaced triangular structures in L5a. It has been reported that the POm projects more axons to L5a neurons vertically aligned under septa than to the adjacent neurons below the barrels $[1,2,7]$. This distinct projection pattern of the POm afferents may recruit their postsynaptic partner L5a pyramidal neurons to form a projection-related distribution pattern. To test this hypothesis, a detailed analysis of the distribution of CRpositive L5a neurons was performed by immunostaining at 


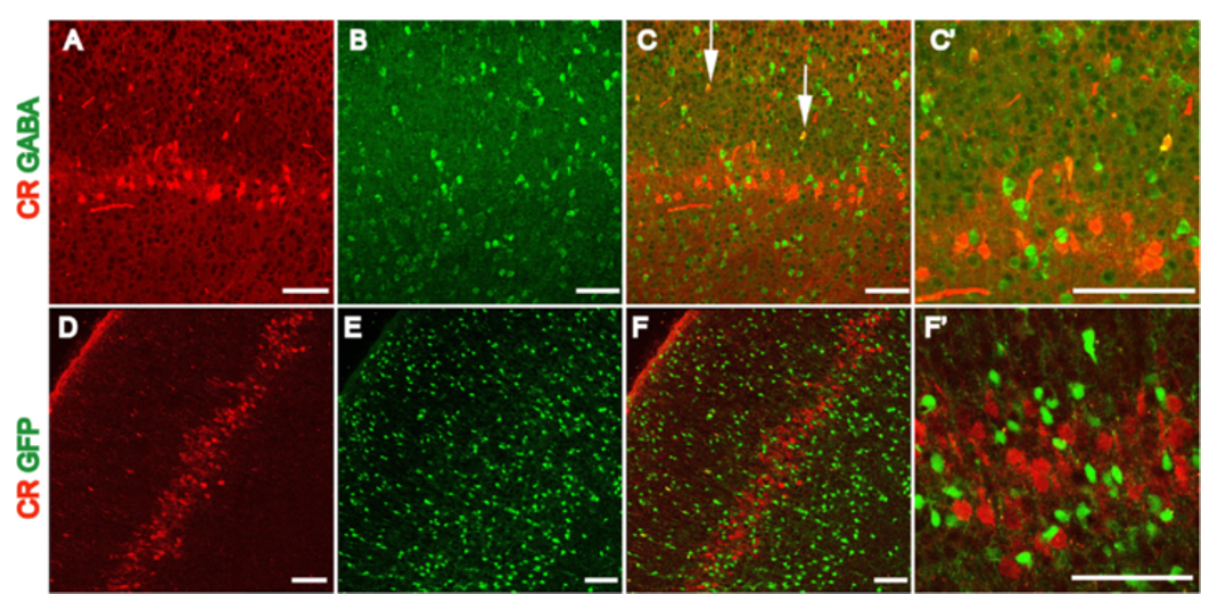

Figure 3 CR-positive neurons in L5a are not GABAergic interneurons. Numerous GABA-positive interneurons are detected in the barrel cortex at P8 (B, C), but none of these neurons are positive for CR in L5a (A-C'). CR- and GABA-positive interneurons can be observed in other areas of the cortex (C, arrows). In the GAD67-GFP transgenic cortex, abundant GFP-positive interneurons are detected, but none of these neurons are positive for $C R$ in $L 5 a\left(\mathbf{E}, \mathbf{F}, \mathbf{F}^{\prime}\right)$. Scale bar: $100 \mu \mathrm{m}$.

various time points during development of the barrel cortex. At P3, the radial processes of L5a neurons started to extend into the overlying L4 (Figure 4A,B). At P4, CR was observed to be expressed in more L5a neurons and formed a clear row-like structure (Figure $4 \mathrm{C}$ ). Furthermore, we found that the CR-positive L5a neurons displayed a nonhomogeneous distribution with some cells aggregated into serrated structures, and the pattern was quite similar to that of their presynaptic POm projections (Figure 4C,D). Regularly spaced CR-positive processes were observed to extend from the serrated structures into L4 (Figure 4D). The expression of CR peaked at P8 (Figure 4I), and the serrated distribution pattern became more defined, with the neuropil spanning all of L4 and forming a prominent septa-like pattern (Figure 4J). The septa-like pattern could be observed until P15, but the expression level of CR in L5a decreased from P8 on (Figure 4K). The structures between the septa-like CR-positive processes might represent prospective barrels (Figure 4I-K). By P30, the CRpositive row-like structure with septa-like processes had almost disappeared, and the barrel structures became more apparent (Figure 4L). These observations suggested that L5a neurons could be recruited by inputs from the POm to form serrated structures with their processes extending to L4 as septa-like structures that closely resembled the projection pattern of the POm into L5a.

It has been reported that the first postnatal week is critical for experience-dependent pattern formation in the barrel cortex. The afferent signal is the principal element in experience-dependent neuronal patterning $[3,22,23]$. It is possible that the distribution pattern of CR-positive L5a neurons may also be input-dependent.
To further detect whether the distribution pattern of CR in L5a depends on sensory input, an early transection of the infraorbital nerve (ION) experiment was carried out. ION transection at P2 led to markedly reduced expression of $\mathrm{CR}$ in the neuropil and cell bodies of the L5a neurons by P3 (Figure 4E,F). By P4, the CR-positive processes that extend to $\mathrm{L} 4$ had become shorter, and the septa-like pattern disappeared (Figure 4G,H). At P8 and P15, few septa-like CR-positive processes were observed to extend to L4. More interestingly, the serrated distribution pattern of CR-positive L5a neurons also disappeared, and only the uniform row-like CR-positive zone remained (Figure 4M,N,O). By P30, expression of CR in L5a could no longer be detected, similar to observations of the control. Taken together, our data indicate that the unique serrated alignment of CR-positive L5a pyramidal neurons is related to the paralemniscal pathway and depends on peripheral sensory input.

\section{The alignment of L5a neurons develops synchronously} with formation of the cortical barrels

Timing of the formation of the distinct serrated alignment of L5a neurons corresponds to the time window of cortical barrel formation and is input dependent, suggesting that the lemniscal and paralemniscal pathways might develop synchronously. We next compared the temporospatial development of the neuronal pattern of L5a with that of the barrels in L4 within the barrel cortex.

Previously, we reported a transgenic mouse line Fzd10-TauLacZ in which the thalamocortical afferents (TCAs) from the VPM are specifically labeled [24]. We 


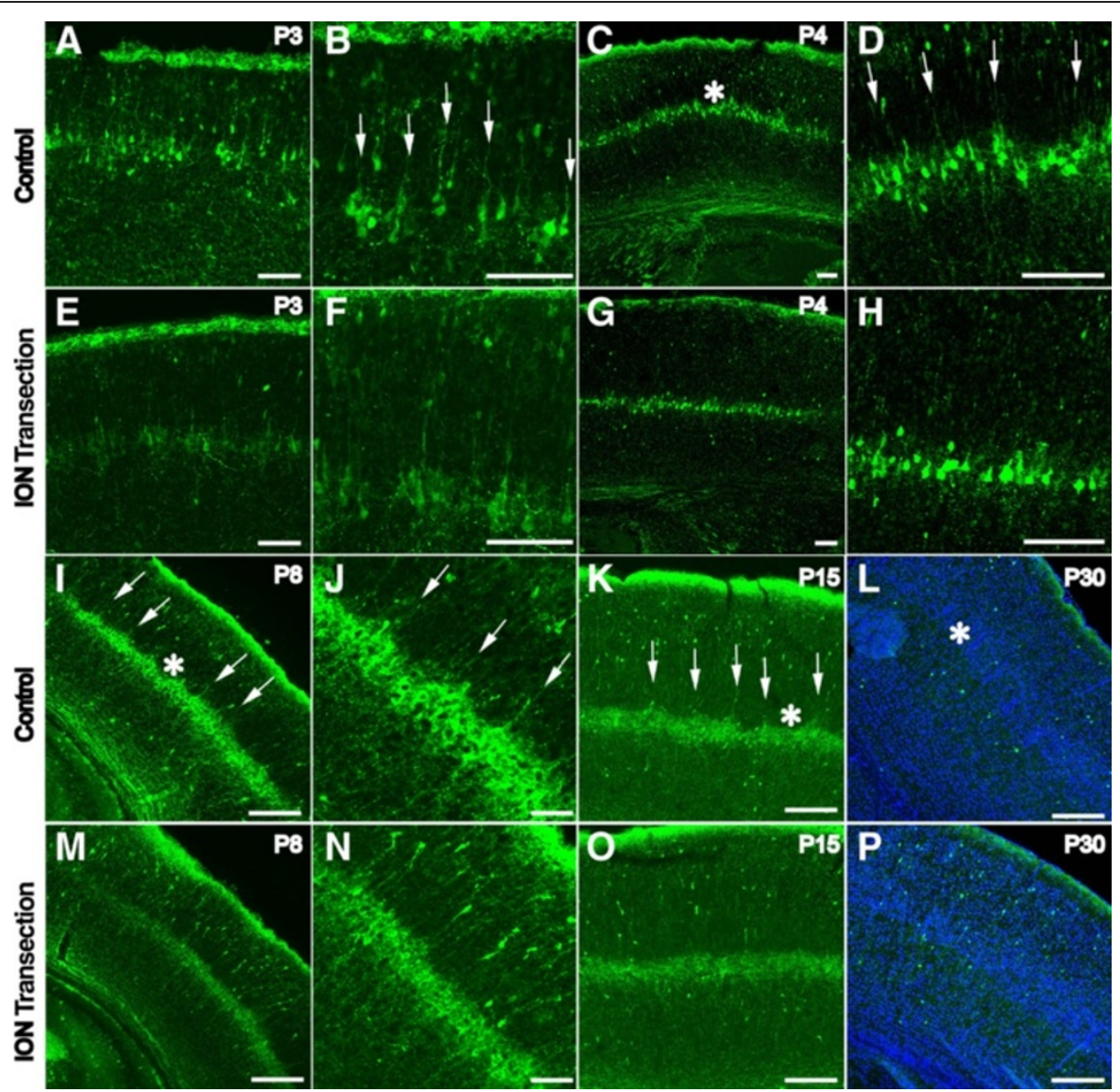

Figure 4 Maintaining the distinct serrated alignment of CR-positive L5a neurons requires sensory input. CR-positive neurons in L5a send long processes into L4 at P3 (A, B, arrows). Until P4, the CR-positive L5a neurons display the distinct serrated aligning pattern, and their processes form septa-like structures (C, D). At P8, the septa-like structures become more distinct (I, J). The expression pattern of CR is maintained until P15 (K). At P30, the CR expression in L5a disappears, and the barrels in L4 become more distinct. After ION transection at P2, the expression level of CR rapidly declines at P3 (E, F). From P4 to P15, the processes of the CR-positive neurons become shorter; the distinct serrated aligning pattern is disrupted; and no septa-like structures of the CR-positive processes are observed $(\mathbf{G}, \mathbf{H}, \mathbf{M}, \mathbf{N}$, and $\mathbf{O})$. By P30, the expression of CR in L5a disappears, and only scattered CR-positive neurons are observed throughout the cortex $(\mathbf{P})$. The asterisks in $\mathbf{C}, \mathbf{I}$ and $\mathbf{L}$ denote barrels and prospective barrels. The arrows in $\mathbf{B}, \mathbf{D}, \mathbf{J}$, and $\mathbf{K}$ denote processes that extend to $L 4$ to form septa-like structures. Scale bar: $100 \mu \mathrm{m}$.

used the same Fzd10 promoter to generate an EGFP reporter mouse line (Figure 5A). Using this mouse line, we found that the GFP-positive TCAs had already reached the deep layer in the barrel cortex at E17.5 (Figure 5B) and had extended their arbors into L4 by P0 (Figure $5 \mathrm{C}$ ). However, at P0, no CR expression was detected in L5a (Figure 5C). By P3 and P4, the GFP-positive TCA terminals had invaded L4 (Figure 5D,E,F) and segregated into clusters, but the clusters were not completely separated from each other (Figure 5E,F). Meanwhile, CR expression was observed in L5a, and some of the CR-positive neuropil had begun to extend into the overlying L4 (Figure 5D,G,H). At P8, the GFP-positive TCA terminals in L4 were completely segregated into distinct clusters to form barrels (Figure 5M,N). At the boundary of L4 and L5, the CR-positive L5a pyramidal neurons intruded into regions between the TCA terminal clusters and formed regularly spaced triangular alignment structures from which the CR-positive neuropil extended into L4 to form a septa-like pattern (Figure 5M,N). The alignment of the CR-positive L5a neurons complemented this pattern of GFP-positive TCA terminals. From P8 to P15, when the barrels were nearly mature, the GFP-positive TCA clusters became denser, and the expression intensity of CR decreased (Figure 5O,P). We then performed ION lesions at P2 and found that the unique alignment of CR-positive neurons was disrupted and that the CR-positive neuropil became shorter. Although the GFP-positive TCAs were able to reach L4, no barrels formed. The serrated pattern of the L5a neurons disappeared; instead, a uniform CR-positive band was detected, and no septa-like 


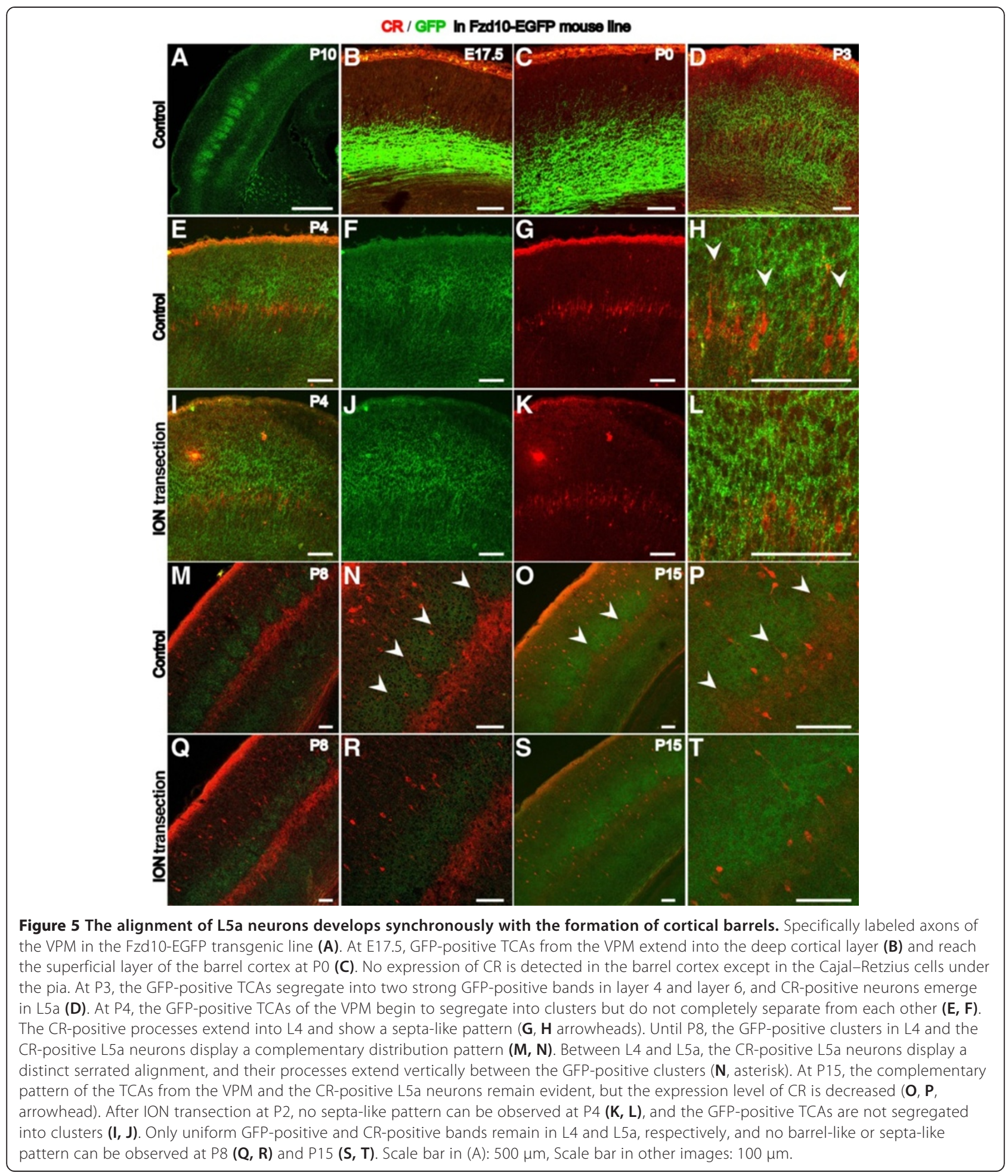

pattern was observed (Figure 5K-L, Q-T). These results suggest that segregation of the VPM projections and formation of the CR-positive L5a pattern may occur in parallel during development of the cortical barrels.
The serrated alignment of L5a neurons is disrupted in CR mutants

The distinct expression pattern of CR in L5a neurons raised the possibility that $\mathrm{CR}$ may be important for 
development of the paralemniscal pathway. As a member of the calmodulin superfamily, CR plays an important role in the precise regulation of intracellular calcium signals and neuronal excitability [13,25-27]. Therefore, we next examined the alignment of L5a neurons in CR knockouts [28]. In situ hybridization was performed to detect the distribution of the ets variant 1 (Etv1) gene, which is a transcription factor reported to be specifically expressed in L5a neurons $[29,30]$. At P8 in controls, Etv1-positive L5a neurons displayed a distinctive serrated pattern with regularly spaced triangular structures protruding towards the overlaying layer that corresponded to the pattern of $\mathrm{CR}$ (Figure 6C and E, arrows). However, in CR mutants, the serrated pattern was disrupted with no triangular structures and a uniform distribution of Etv1positive L5a neurons (Figure 6D-F), indicating that CR is important for the formation of the distinct serrated arrangement of L5a pyramidal neurons.

\section{CR is also specifically expressed in the relay nucleus of the paralemniscal pathway}

The paralemniscal pathway originates from large neurons in the interpolar subnucleus of the spinal trigeminal nucleus (Sp5i), which project to the POm in the thalamus [31], and these POm afferents target L1, L5a and the septum-related L4 neurons in the S1 cortex. We found that, in addition to being expressed in L5a pyramidal neurons, $\mathrm{CR}$ was also dynamically expressed in the POm and Sp5i, which are the relay nuclei of the paralemniscal pathway. At E14.5, the expression of CR could be observed in the POm, which is located lateral to the parafascicular thalamic nucleus (PF) (Figure 7A) and the Sp5i (Figure 7G). This expression became stronger at
E16.5 (Figure 7B,H) and continued to persist (Figure 7CF,I-L). To determine whether CR-positive neurons are excitatory neurons or interneurons, double labeling of anti-CR and GFP was performed in the GAD67-GFP knock-in mouse line at P8, when the pattern of the paralemniscal pathway had formed in L5a. No co-localization was detected in the POm or Sp5i (Figure 8A-F, insert), which indicates that these CR-positive neurons are excitatory projection neurons. The expression of $\mathrm{CR}$ in the developing paralemniscal pathway suggests that CR may be involved in development and maturation of the paralemniscal pathway as a modulator of neuronal excitability and of calcium signaling pathways.

\section{Discussion}

In rodents, the barrel cortex is a unique columnar neuronal circuit with barrel and septa columns (circuit) that receive distinct sensory information through the lemniscal and the paralemniscal pathways from the VPM and POm, respectively $[1,2,5]$. Information from both circuits must be integrated in the barrel cortex to mediate precise object recognition and related behavioral tasks [1]. It has been reported that barrel dysfunction is associated with Fragile $\mathrm{X}$ mental retardation syndrome [32] and Huntington's disease [33]. Therefore, it is crucial to understand the mechanisms underlying the development of lemniscal and paralemniscal pathways, as well as the integration of information in the whisker-barrel cortex.

In the barrel cortex, L5a pyramidal neurons are of particular interest not only because they receive subcortical inputs exclusively from the paralemniscal pathway but also because they are involved in both the barrel and septa circuits $[4,9,11,12]$. L5a pyramidal neurons are considered to be an important integration site of the

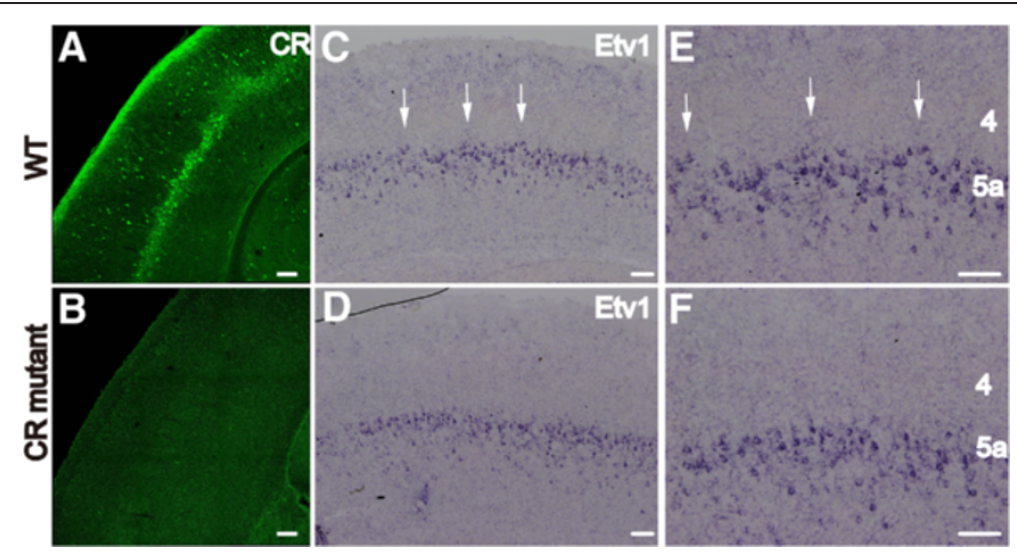

Figure 6 The distinct serrated alignment of L5a pyramidal neurons is disrupted in the CR KO cortex. CR is effectively deleted in the CR mutant cortex compared to that of the WT at P8 (A, B). In situ hybridization for the transcription factor Etv1, which is mainly expressed in the L5a neurons, reveals a prominent serrated alignment with regularly spaced triangular structures protruding towards the overlaying layer in WT animals (C, E, arrows) that is disrupted in the mutants (D, F). Scale bar: $100 \mu \mathrm{m}$. 


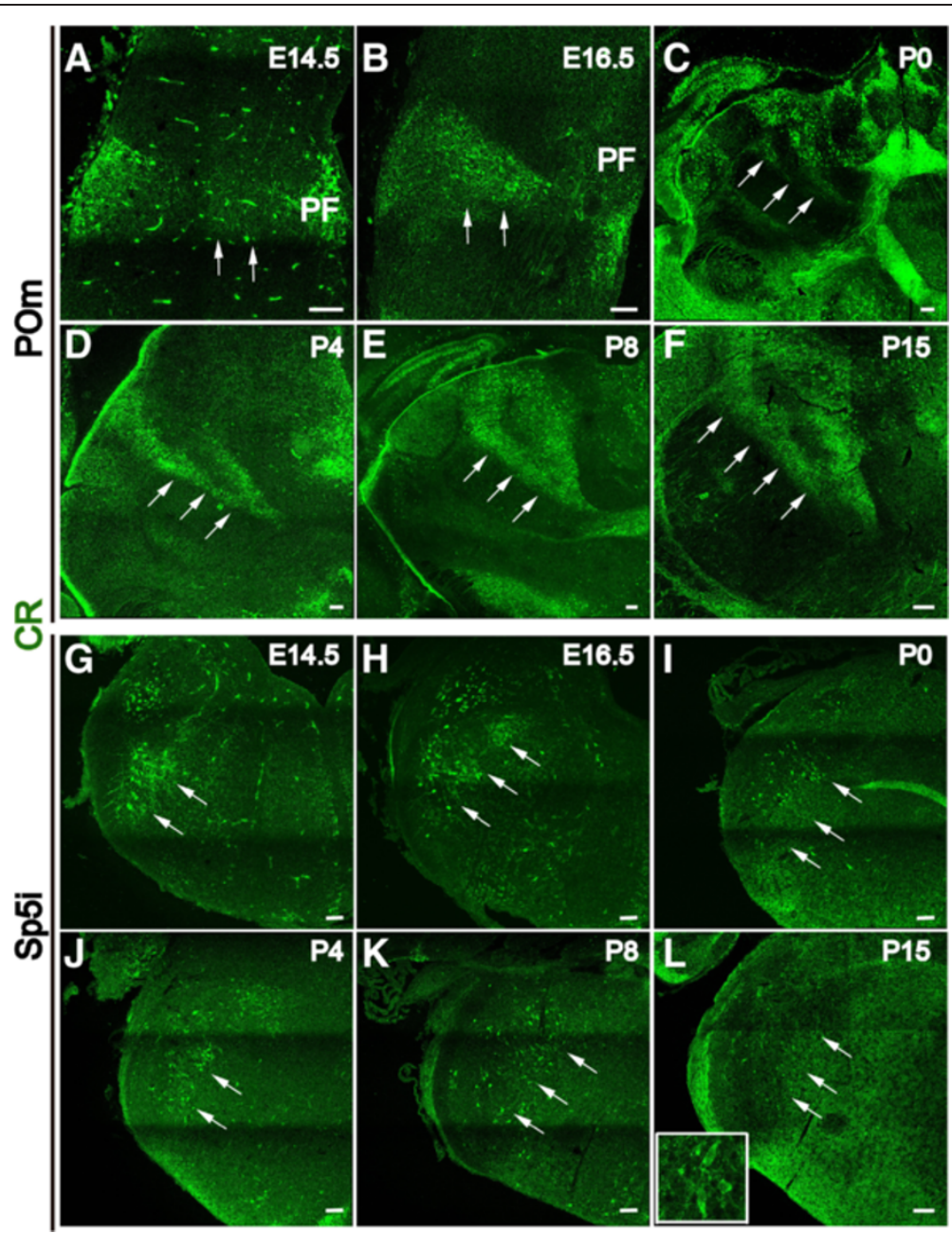

Figure 7 CR is strongly expressed in the developing paralemniscal pathway. At E14.5, weak CR can be observed in the POm, lateral to the parafascicular thalamic nucleus (A, arrows). At E16.5, the expression of CR in the POm becomes stronger (B, arrows). After birth, CR is continuously expressed in the POm at least through P15 (C-F, arrows). Strong expression of CR is also observed in Sp5i, which is the relay nucleus of the paralemniscal pathway, from E14.5 to P15 (G-L, arrows). Scale bar: $100 \mu \mathrm{m}$.

lemniscal and paralemniscal pathways and the key output layer of the barrel cortex [5,10,12,34]. However, information about L5a pyramidal neurons has remained limited.

The unique alignment of L5a pyramidal neurons may form part of the morphological basis for information integration in the barrel cortex

It is well known that the formation of peripheral-inputrelated neuronal patterning in the barrel cortex depends on thalamocortical afferents. Previous studies have demonstrated that the neuronal pattern in the lemniscal pathway in L4 is organized only after segregation of the presynaptic afferents [3,22]. However, it remains unclear whether L5a neurons in the paralemniscal pathway integrate into an input-dependent distribution pattern during development that resembles the pattern of L4 neurons.
Using retrograde neuronal tracing, POm axons have been shown to project mainly to L5a. Projections of the POm to L5a neurons in septa columns are denser than those to their neighbors just below the barrel columns, and POm projections form a row-like pattern with regularly spaced triangular structures, indicating that there may be a postsynaptic paralemniscal input-related neuronal pattern in L5a. In this study, using CR as a specific marker for L5a pyramidal neurons, we determined that L5a neurons are organized in a serrated alignment pattern complementary to the distribution pattern of the POm projection terminals. Furthermore, the disruption of this unique alignment after ION transection suggests that it is input dependent. Taken together, in the barrel cortex, there is an input-dependent neuronal pattern in L5a neurons of the paralemniscal pathway comparable to that formed by L4 neurons in the lemniscal pathway. 


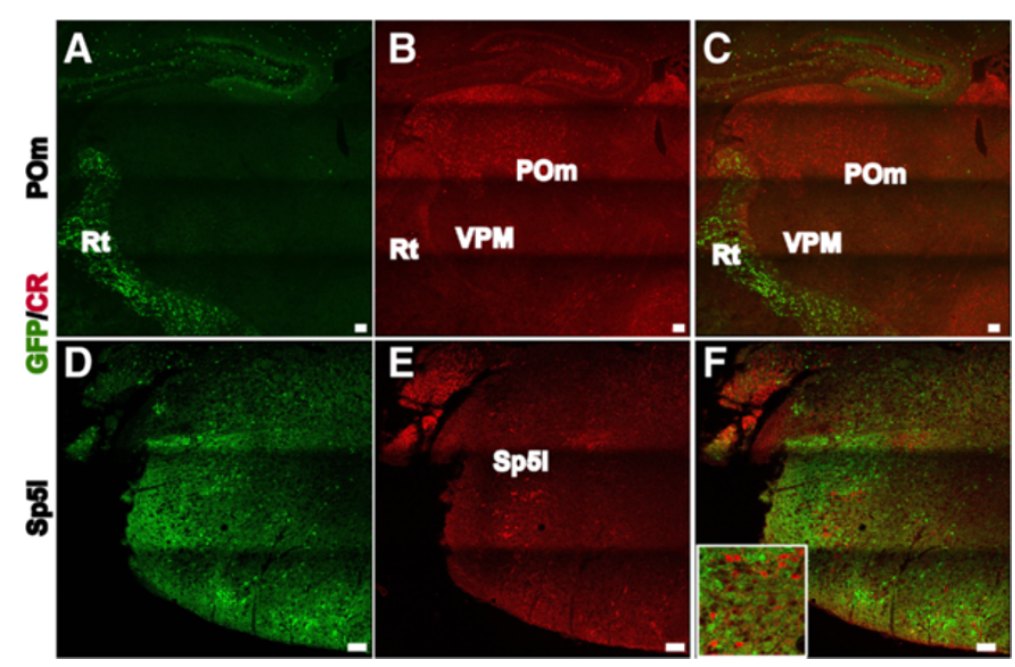

Figure 8 CR-positive neurons in the Sp5i and POm are not GABAergic interneurons. Double immunolabeling of anti-CR and GFP in GAD67-GFP mice at P8 indicates that GFP-positive neurons are located in the reticular thalamic nucleus (Rt) and are not co-labeled with CR (A). CR expression is specifically detected in the POm in the dorsal thalamus (B, C). In the medulla oblongata, CR-positive neurons and GFP-positive neurons can be detected in the Sp5i, but CR and GFP are not co-labeled (D-F). The insert represents an amplification of CR-positive neurons in the Sp5i. Scale bar: $100 \mu \mathrm{m}$.

Although L5a pyramidal neurons are the main targets of the paralemniscal pathway, they also receive indirect lemniscal pathway input through monosynaptic connections between L4 barrel neurons and L5a neurons [9], as well as intracolumnar input from L2/3 and L5b in barrel columns [8,35]. L5a neurons also send weak outputs to L2/3 neurons in the barrel columns $[4,9,10,35,36]$. In addition, L5a pyramidal neurons form local connections [11], sending outputs to intracolumn L5b cells [37], intercortical motor and secondary somatosensory cortices $[5,10,34]$, and the basal ganglia [38,39]. Electrophysiological studies have unanimously indicated the importance of L5a pyramidal neurons as the integration site of the lemniscal and paralemniscal pathways. Feldmeyer, Roth and colleagues have reported synaptic connections between L4 spiny stellate cells and L5a pyramidal cells, and postsynaptic L5a somata are mostly located under the barrel wall and septa, suggesting the existence of a short circuit between the lemniscal and paralemniscal pathways [9]. They also found local connections in paired L5a pyramidal neurons, mainly along the lateral border of barrel columns, and these connections appear to form vertical clusters. It is plausible that the vertical clusters may correspond to the extrusions within the serrated pattern of L5a pyramidal neurons observed in this study. Our observations have provided morphological support for connections between the barrel and septa columns. Meanwhile, more attention should be paid to the L5a neuronal intrusions into the lower part of L4 for further studies of the paralemniscal-related neuronal circuit and the integration of information in the barrel cortex.
The long processes of CR-positive L5a neurons may contribute to the composition of septa

In this study, we have shown that development of the L5a neuronal pattern parallels the segregation of VPM axons during the time window of barrel formation, indicating that the lemniscal and paralemniscal pathways may interact to regulate pattern formation and refinement of the barrel cortex.

In the somatosensory cortex, L4 is organized into celldense barrels and cell-sparse septa [40], which receive thalamocortical input from the VPM and POm, respectively $[1,2]$. However, because septa are small and contain few cells, their properties remain largely unknown. In this study, from intrusions of L5a neuron alignment, we directly observed that the CR-positive neuropil extends into L4 and forms a septa-like pattern, which suggests that the dendrites of L5a pyramidal neurons may be components of the septa. Further studies are needed to understand the connections of septa with L5a pyramidal neurons and to help elucidate the function of septa and the septal circuit.

CR may be involved in development of the paralemniscal pathway and formation of the L5a neuronal alignment

The dynamic expression of CR in L5a neurons while cortical barrels are developing and the disruption of the distinct alignment of L5a pyramidal neurons in CR-null mutants indicate that $\mathrm{CR}$, a critical element of calcium signaling, is involved in the development and maintenance of input-dependent patterning in L5a pyramidal neurons. Furthermore, the specific expression of CR in the paralemniscal pathway indicates that $\mathrm{CR}$ may also be required 
during development of the paralemniscal pathway and may therefore be important for its function. In this study, we were unable to evaluate the electrophysiological characteristics of CR-deficient L5a neurons. Further studies using CR mutants will be useful to elucidate the mechanisms underlying development of the paralemniscal pathway and its interaction with the lemniscal pathway in the barrel cortex.

\section{Conclusions}

In this study, we have shown that, as a main target of the paralemniscal pathway in the barrel cortex, CR-positive L5a pyramidal neurons form the POm projection-related neuronal pattern during early postnatal development, synchronously with lemniscal-related barrel formation. The expression of $\mathrm{CR}$ in the paralemniscal pathway and the changed patterning of the L5a neurons in the CR mutant indicated that CR may be important for development of the paralemniscal pathway and the barrel cortex. Our data provide an important morphological basis for studying the functions of L5a pyramidal neurons and development of the paralemniscal pathway in the barrel cortex.

\section{Methods}

\section{Animals}

The generation of GAD67-GFP knock-in mice has been previously described [21]. The Calretinin knockout line [28] was purchased from Jackson Laboratory. The Fzd10EGFP transgenic line was generated using the Frizzled10 promoter to drive the reporter gene as previously reported [24]. Both male and female animals were used in this study. The day the vaginal plug was detected was designated as embryonic day 0.5 (E0.5). The day of birth was designated as postnatal day 0 (P0). Each group subjected to specific examination included at least 3 mice. All of the animals were bred in the animal facility at Southeast University. All of the experiments were performed according to the guidelines approved by Southeast University.

\section{ION transection}

The pups were anesthetized by hypothermia at P2. Under a stereoscopic microscope, the mystacial whiskers on the left side were plucked before the operation. A small incision was made just behind the left whisker pad. The infraorbital nerve was exposed and transected with ophthalmic forceps. After hemostasis and saturation, the pups were put on a heating blanket to recover and returned to their home cages. The pups were then harvested at P3, P4, P8, P15 and P30, respectively. Each time point contained at least 5 pairs of animals.

\section{Tissue preparation}

Mice up to P4 were anesthetized by hypothermia. Mice at P8, P15, and P30 were anesthetized with sodium pentobarbital ( $50 \mathrm{mg} / \mathrm{kg}$ body weight) via intraperitoneal injection. Each anesthetized mouse was perfused transcardially with $0.1 \mathrm{M}$ phosphate-buffered saline (PBS, $\mathrm{pH}$ 7.4) followed by $4 \%$ paraformaldehyde (PFA) in $0.1 \mathrm{M} \mathrm{PBS}$ under the stereoscopic microscope. The brains were removed, post-fixed for 8-10 hours in 4\% PFA and protected with $30 \%$ sucrose in $0.1 \mathrm{M}$ PBS overnight at $4^{\circ} \mathrm{C}$. The brains were sectioned in the coronal plane using a freezing microtome (Leica). The thicknesses of the sections were $16 \mu \mathrm{m}$ (up to P4) and $25 \mu \mathrm{m}$ (P8, P15 and P30).

\section{Immunostaining}

Immunostaining was performed according to standard protocols [41]. Briefly, the sections were permeabilized in PBS containing $0.1 \%$ Triton X-100 (PBT) for $30 \mathrm{~min}$, blocked with $10 \%$ calf serum in PBT for $2 \mathrm{~h}$, incubated at $4^{\circ} \mathrm{C}$ with primary antibody overnight, then incubated with the secondary antibody for $2 \mathrm{~h}$ at room temperature (RT). Slices were then counterstained with the fluorescent nuclear stain DAPI and mounted under coverslips. The sections were washed with $0.1 \mathrm{M}$ PBS between the staining steps.

\section{BrdU birth-dating}

BrdU (Sigma-Aldrich, B5002) was dissolved in physiological saline at a concentration of $10 \mathrm{mg} / \mathrm{ml}$. Pregnant mice were given $\mathrm{BrdU}(50 \mathrm{mg} / \mathrm{kg}$, intraperitoneal injections) at E12.5, E13.5, E14.5, E15.5, and brains of the offspring were harvested at P8 for birth-dating analysis. CR immunostaining was carried out first, and sections were re-fixed with $4 \%$ PFA for $20 \mathrm{~min}$, then treated with $2 \mathrm{~N}$ $\mathrm{HCl}$ for $15 \mathrm{~min}$ at $42^{\circ} \mathrm{C}$. After $\mathrm{HCl}$ was washed out with PBS, immunostaining for BrdU was applied according to the standard procedure described above. At least three brains were collected from independent litters at each time point.

\section{Primary and secondary antibodies}

The following antibodies were used: rabbit polyclonal anti-CR (Chemicon, AB5054, 1:1000), mouse anti-CR (Chemicon, MAB1568, 1:1000), rat polyclonal anti-BrdU (Abcam, ab6326, 1:1000), rabbit polyclonal anti-Cux1 (Santa Cruz, sc-13024, 1:500), rat polyclonal anti-Ctip2 (Abcam, ab18465, 1:1000), rabbit polyclonal anti-Foxp2 (Abcam, ab16046, 1:1000), rabbit polyclonal antiNeuroD2 (Abcam, ab31938, 1:100), rabbit polyclonal anti-GABA (Chemicon, AB131, 1:500), chicken anti-GFP (Invitrogen, A10262, 1:500). FITC goat anti-mouse IgG (Jackson ImmunoResearch, 115-095-146, 1:200), Alexa Fluor 555 donkey anti-mouse IgG (Invitrogen, A31570, 1:500), Alexa Fluor 633 goat anti-mouse IgG (Invitrogen, A21050, 1:500), Alexa Fluor 488/633 goat anti-rabbit IgG (Invitrogen, A11008/A21071, 1:500), Alexa Fluor 
550 goat anti-rat IgG (Invitrogen, A11006, 1:500), and Alexa Fluor 488 conjugated AffiniPure donkey antichicken IgG (Jackson ImmunoResearch, 1:1000). DAPI was purchased from Sigma-Aldrich (D9564).

\section{Microscopic imaging}

Sections were imaged under a confocal microscope (FV1000, Olympus) using the 405, 488, 559, and 633 laser channels. Pictures were captured sequentially for each channel under the $10 \times, 20 \times$ and $40 \times$ objectives with excitation lasers and detection spectra calibrated to minimize channel crosstalk. The image format was $1024 \times 1024$ pixels. Confocal image stacks were analyzed using FV10-ASW 4.1 Viewer.

\section{In situ hybridization}

Brains from P8 mice were perfused with 4\% PFA, postfixed in 4\% PFA overnight, and cryoprotected in 30\% sucrose/DEPC-PBS at $4^{\circ} \mathrm{C}$. Coronal sections $(25 \mu \mathrm{m}$ thick) were sliced with a Leica CM 3050S cryostat and stored at $-70^{\circ} \mathrm{C}$ until use. For Etv1 probe preparation, total RNA was extracted from E14.5 brains. Using primers with the sequences GAG TCA CAG GCC AGT AGC ATT and ACA GCA GCG GAA GCA TTA G, we amplified Etv1 fragments that were approximately $421 \mathrm{bp}$ in length (2380-2801), and these fragments were subcloned into pBluescript SK vectors and linearized (details available upon request). In situ hybridization was performed as described previously [41]. Pictures were captured with an OlympusBX61 microscope (Tokyo, Japan). Three brain pairs from three independent litters were examined.

\section{Cell counting}

Coronal sections at three comparative levels from the anterior to posterior in each brain were examined. Pictures were acquired using an Olympus FluoView FV1000 confocal microscopy with the $20 \times$ objective lens. Cell counting was performed on sections using image Pro Plus software (Media Cybernetics).

\section{Abbreviations}

ION: Infraorbital nerve; Pr5: Principal sensory trigeminal nucleus; VPM: The contralateral ventroposteromedial nucleus of the thalamus; Sp5i: The interpolar subnucleus of the spinal trigeminal nucleus; POm: The contralateral posterior medial nucleus; L4: Layer 4; P4: Postnatal day 4; E12.5: Embryonic day 12.5; CR: Calretinin; Cux1: Cux family transcription factor 1; Ctip2: Chicken ovalbumin upstream promoter transcription factor-interacting protein 2; NeuroD2: Transcription factor neurogenic differentiation factor 2; Foxp2: Forkhead box protein 2; GABA: Gamma-aminobutyric acid; GAD67: 67-kDa glutamic acid decarboxylase; GFP: Green fluorescent protein; Fzd10: Frizzled10; EGFP: Enhanced green fluorescent protein; TCAs: Thalamocortical afferents; ETV1: Ets variant 1; PF: Parafascicular thalamic nucleus; Rt: Reticular thalamic nucleus.
}

\section{Competing interests}

The authors declare that they have no competing interests.

\section{Authors' contributions}

$J L$ and $C Z$ designed the study; $J L$ performed immunostaining and cell counting; BL generated the Fzd10-EGFP transgenic line, $X Z$ and BY performed in situ hybridization; BY, WG and KW performed genotyping and the birth-dating test; $Y Y$ and $Y G$ performed the infraorbital nerve transection; YY and SW provided the GAD76-GFP knock-in mouse line; JL, XW and CZ analyzed the data and wrote the paper. All authors read and approved the final manuscript.

\section{Authors' information}

$J$ has been a lecturer at the Department of Anatomy and Neuroscience, Medical School at Southeast University since 1998, teaching systemic and regional anatomy. From 2000 to 2004, JL studied the pathogenesis of cerebrovascular disease and earned her M.S. Since 2008, JL has studied as a Ph.D. student in the Key Laboratory of Developmental Genes and Human Diseases, MOE, Southeast University, working on neurodevelopment and related disorders with a particular focus on the primary development and function of the barrel cortex.

\section{Acknowledgments}

We thank Professor Yongchun Yu (Institutes of Brain Science, Fudan University) for his suggestions, Yiquan Wei and Li Liu for their assistance with laboratory and animal care and other members of the laboratory for their discussions. This work was supported by grant 2012AA022402 from the National High Technology Research and Development Program of China and grants 91232301 and 31171040 from the National Natural Science Foundation of China to CZ.

\section{Author details}

${ }^{1}$ Key Laboratory of Developmental Genes and Human Diseases, MOE, Department of Anatomy and Neuroscience, Medical School, Southeast University, Nanjing 210009, PR China. ${ }^{2}$ Center of Depression, Beijing Institute for Brain Disorders, Beijing 100069, PR China. ${ }^{3}$ Institute of Neurobiology, Institutes of Brain Science and State Key Laboratory of Medical Neurobiology, Fudan University, Shanghai 200032, PR China. ${ }^{4}$ Department of Genetic and Behavioral Neuroscience, Gunma University Graduate School of Medicine, 3-39-22 Showa-machi, Maebashi 371-8511, Japan. ${ }^{5}$ Department of Anatomy, Histology and Embryology, K.K. Leung Brain Research Centre, School of Basic Medicine, Fourth Military Medical University, Xi'an 710032, PR China.

Received: 28 June 2014 Accepted: 4 November 2014

Published online: 18 November 2014

\section{References}

1. Alloway KD: Information processing streams in rodent barrel cortex: the differential functions of barrel and septal circuits. Cereb Cortex 2008, 18:979-989.

2. Lu SM, Lin RC: Thalamic afferents of the rat barrel cortex: a light- and electron-microscopic study using Phaseolus vulgaris leucoagglutinin as an anterograde tracer. Somatosens Mot Res 1993, 10:1-16.

3. Erzurumlu RS, Gaspar P: Development and critical period plasticity of the barrel cortex. Eur J Neurosci 2012, 35:1540-1553.

4. Bureau I, von Saint PF, Svoboda K: Interdigitated paralemniscal and lemniscal pathways in the mouse barrel cortex. PLoS Biol 2006, 4:e382.

5. Feldmeyer D: Excitatory neuronal connectivity in the barrel cortex. Front Neuroanat 2012, 6:24.

6. Lubke J, Feldmeyer D: Excitatory signal flow and connectivity in a cortical column: focus on barrel cortex. Brain Struct Funct 2007, 212:3-17.

7. Wimmer VC, Bruno RM, de Kock CP, Kuner T, Sakmann B: Dimensions of a projection column and architecture of VPM and POm axons in rat vibrissal cortex. Cereb Cortex 2010, 20:2265-2276.

8. Schubert D, Kotter R, Luhmann HJ, Staiger JF: Morphology, electrophysiology and functional input connectivity of pyramidal neurons characterizes a genuine layer va in the primary somatosensory cortex. Cereb Cortex 2006, 16:223-236.

9. Feldmeyer D, Roth A, Sakmann B: Monosynaptic connections between pairs of spiny stellate cells in layer 4 and pyramidal cells in layer $5 \mathrm{~A}$ indicate that lemniscal and paralemniscal afferent pathways converge in the infragranular somatosensory cortex. J Neurosci 2005, 25:3423-3431.

10. Larsen DD, Wickersham IR, Callaway EM: Retrograde tracing with recombinant rabies virus reveals correlations between projection targets 
and dendritic architecture in layer 5 of mouse barrel cortex. Front Neural Circuits 2007, 1:5.

11. Frick A, Feldmeyer D, Helmstaedter M, Sakmann B: Monosynaptic connections between pairs of L5A pyramidal neurons in columns of juvenile rat somatosensory cortex. Cereb Cortex 2008, 18:397-406.

12. Shepherd GM, Svoboda K: Laminar and columnar organization of ascending excitatory projections to layer $2 / 3$ pyramidal neurons in rat barrel cortex. J Neurosci 2005, 25:5670-5679.

13. Camp AJ, Wijesinghe R: Calretinin: modulator of neuronal excitability. Int J Biochem Cell Biol 2009, 41:2118-2121.

14. Melvin NR, Dyck RH: Developmental distribution of calretinin in mouse barrel cortex. Brain Res Dev Brain Res 2003, 143:111-114.

15. Caputi A, Rozov A, Blatow M, Monyer H: Two calretinin-positive GABAergic cell types in layer $2 / 3$ of the mouse neocortex provide different forms of inhibition. Cereb Cortex 2009, 19:1345-1359.

16. Barinka F, Druga R: Calretinin expression in the mammalian neocortex: a review. Physiol Res 2010, 59:665-677.

17. Chen B, Wang SS, Hattox AM, Rayburn H, Nelson SB, McConnell SK: The Fezf2-Ctip2 genetic pathway regulates the fate choice of subcortical projection neurons in the developing cerebral cortex. Proc Natl Acad SCi U S A 2008, 105:11382-11387.

18. Cubelos B, Sebastian-Serrano A, Beccari L, Calcagnotto ME, Cisneros E, Kim S, Dopazo A, Alvarez-Dolado M, Redondo JM, Bovolenta P, Walsh CA, Nieto M: Cux1 and Cux2 regulate dendritic branching, spine morphology, and synapses of the upper layer neurons of the cortex. Neuron 2010, 66:523-535.

19. Ince-Dunn G, Hall BJ, Hu SC, Ripley B, Huganir RL, Olson JM, Tapscott SJ, Ghosh A: Regulation of thalamocortical patterning and synaptic maturation by NeuroD2. Neuron 2006, 49:683-695.

20. Hisaoka T, Nakamura Y, Senba E, Morikawa Y: The forkhead transcription factors, Foxp1 and Foxp2, identify different subpopulations of projection neurons in the mouse cerebral cortex. Neuroscience 2010, 166:551-563.

21. Tamamaki N, Yanagawa Y, Tomioka R, Miyazaki J, Obata K, Kaneko T: Green fluorescent protein expression and colocalization with calretinin, parvalbumin, and somatostatin in the GAD67-GFP knock-in mouse. J Comp Neurol 2003, 467:60-79.

22. Sehara K, Kawasaki H: Neuronal circuits with whisker-related patterns. Mol Neurobiol 2011, 43:155-162.

23. Li H, Crair MC: How do barrels form in somatosensory cortex? Ann N Y Acad Sci 2011, 1225:119-129.

24. Zhao C, Guan W, Pleasure SJ: A transgenic marker mouse line labels Cajal-Retzius cells from the cortical hem and thalamocortical axons. Brain Res 2006, 1077:48-53.

25. Gall D, Roussel C, Susa I, D’Angelo E, Rossi P, Bearzatto B, Galas MC, Blum D, Schurmans S, Schiffmann SN: Altered neuronal excitability in cerebellar granule cells of mice lacking calretinin. J Neurosci 2003, 23:9320-9327.

26. Schiffmann SN, Cheron G, Lohof A, d'Alcantara P, Meyer M, Parmentier M, Schurmans S: Impaired motor coordination and Purkinje cell excitability in mice lacking calretinin. Proc Natl Acad Sci U S A 1999, 96:5257-5262.

27. Schwaller B: Cytosolic Ca2+ buffers. Cold Spring Harb Perspect Biol 2010, 2:a004051.

28. Schurmans S, Schiffmann SN, Gurden H, Lemaire M, Lipp HP, Schwam V, Pochet R, Imperato A, Bohme GA, Parmentier M: Impaired long-term potentiation induction in dentate gyrus of calretinin-deficient mice. Proc Natl Acad Sci U S A 1997, 94:10415-10420.

29. O'Connor DH, Huber D, Svoboda K: Reverse engineering the mouse brain. Nature 2009, 461:923-929.

30. Gong S, Doughty M, Harbaugh CR, Cummins A, Hatten ME, Heintz N, Gerfen CR: Targeting Cre recombinase to specific neuron populations with bacterial artificial chromosome constructs. J Neurosci 2007, 27:9817-9823.

31. Veinante $P$, Lavallee $P$, Deschenes $M$ : Corticothalamic projections from layer 5 of the vibrissal barrel cortex in the rat. J Comp Neurol 2000 424:197-204.

32. Galvez R, Gopal AR, Greenough WT: Somatosensory cortical barrel dendritic abnormalities in a mouse model of the fragile $X$ mental retardation syndrome. Brain Res 2003, 971:83-89.

33. Mazarakis NK, Cybulska-Klosowicz A, Grote H, Pang T, Van Dellen A, Kossut M, Blakemore C, Hannan AJ: Deficits in experience-dependent cortical plasticity and sensory-discrimination learning in presymptomatic Huntington's disease mice. J Neurosci 2005, 25:3059-3066.
34. Mao T, Kusefoglu D, Hooks BM, Huber D, Petreanu L, Svoboda K: Long-range neuronal circuits underlying the interaction between sensory and motor cortex. Neuron 2011, 72:111-123.

35. Lefort S, Tomm C, Floyd Sarria JC, Petersen CC: The excitatory neuronal network of the $\mathrm{C} 2$ barrel column in mouse primary somatosensory cortex. Neuron 2009, 61:301-316.

36. Xu X, Callaway EM: Laminar specificity of functional input to distinct types of inhibitory cortical neurons. J Neurosci 2009, 29:70-85.

37. Schubert D, Staiger JF, Cho N, Kotter R, Zilles K, Luhmann HJ: Layer-specific intracolumnar and transcolumnar functional connectivity of layer $\mathrm{V}$ pyramidal cells in rat barrel cortex. J Neurosci 2001, 21:3580-3592.

38. Hoffer ZS, Arantes HB, Roth RL, Alloway KD: Functional circuits mediating sensorimotor integration: quantitative comparisons of projections from rodent barrel cortex to primary motor cortex, neostriatum, superior colliculus, and the pons. J Comp Neurol 2005, 488:82-100.

39. Alloway KD, Crist J, Mutic JJ, Roy SA: Corticostriatal projections from rat barrel cortex have an anisotropic organization that correlates with vibrissal whisking behavior. J Neurosci 1999, 19:10908-10922.

40. Woolsey TA, Van der Loos H: The structural organization of layer IV in the somatosensory region (SI) of mouse cerebral cortex. The description of a cortical field composed of discrete cytoarchitectonic units. Brain Res 1970, 17:205-242

41. Tian C, Gong Y, Yang Y, Shen W, Wang K, Liu J, Xu B, Zhao J, Zhao C: Foxg1 has an essential role in postnatal development of the dentate gyrus. J Neurosci 2012, 32:2931-2949.

\section{doi:10.1186/s13041-014-0084-8}

Cite this article as: Liu et al.: Calretinin-positive L5a pyramidal neurons in the development of the paralemniscal pathway in the barrel cortex. Molecular Brain 2014 7:84.

\section{Submit your next manuscript to BioMed Central and take full advantage of:}

- Convenient online submission

- Thorough peer review

- No space constraints or color figure charges

- Immediate publication on acceptance

- Inclusion in PubMed, CAS, Scopus and Google Scholar

- Research which is freely available for redistribution 